\title{
Exogenous ascorbic acid enhances vitrification survival of porcine in vitro-developed blastocysts but fails to improve the in vitro embryo production outcomes
}

A. Nohalez, C. A. Martinez, I. Parrilla, J . Roca, M. A. Gil, Heriberto RodriguezMartinez, E. A. Martinez and C. Cuello

The self-archived postprint version of this journal article is available at Linköping University Institutional Repository (DiVA):

http:// urn.kb.se/ resolve?urn=urn:nbn:se:liu:diva- 147897

N.B.: When citing this work, cite the original publication.

Nohalez, A., Martinez, C. A., Parrilla, I., Roca, J., Gil, M. A., Rodriguez-Martinez, H., Martinez, E. A., Cuello, C., (2018), Exogenous ascorbic acid enhances vitrification survival of porcine in vitrodeveloped blastocysts but fails to improve the in vitro embryo production outcomes, Theriogenology, 113, 113-119. https:// doi.org/ 10.1016/j.theriogenology.2018.02.014

Original publication available at:

https:// doi.org/10.1016/j.theriogenology.2018.02.014

Copyright: Elsevier

http:// www.elsevier.com/

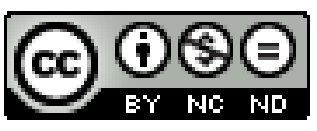


3 Exogenous ascorbic acid enhances vitrification survival of porcine in vitro4 developed blastocysts but fails to improve the in vitro embryo production 5 outcomes

7 A. Nohalez ${ }^{\mathrm{a}, \mathrm{b}}$, C.A. Martinez ${ }^{\mathrm{a}, \mathrm{b}}$, I. Parrilla ${ }^{\mathrm{a}, \mathrm{b}}$, J. Roca ${ }^{\mathrm{a}, \mathrm{b}}$, M.A. Gil ${ }^{\mathrm{a}, \mathrm{b}}$, H. Rodriguez8 Martinez ${ }^{\mathrm{c}}$, E.A. Martinez ${ }^{\mathrm{a}, \mathrm{b}^{*}}$, C. Cuello $\mathrm{a}^{\mathrm{a}, \mathrm{b}}$

10 a Faculty of Veterinary Medicine, International Excellence Campus for Higher 11 Education and Research “Campus Mare Nostrum”, University of Murcia, Murcia, Spain

12 ' Institute for Biomedical Research of Murcia (IMIB-Arrixaca), Murcia, Spain

13 'Department of Clinical \& Experimental Medicine (IKE), Linköping University, 14 Linköping, Sweden

$16 *$ Corresponding author

17 Emilio A. Martinez

18 Facultad de Veterinaria. Campus de Espinardo, 30100, Murcia, Spain

19 E-mail: emilio@um.es

20 Tel.: +34 868884734

21 Fax: +34 868887069 


\section{Abstract}

2 In this study, the effects of addition of the antioxidant ascorbic acid (AsA) were

3 evaluated during porcine in vitro embryo production (IVP) and vitrification. In

4 experiment 1 , the effects of AsA supplementation during IVM, IVF and IVC were

5 evaluated, using a total of 2,744 oocytes in six replicates. The IVM, IVF and embryo

6 IVC media were supplemented or not (control) with $50 \mu \mathrm{g} / \mathrm{mL}$ AsA in all possible

7 combinations. No significant effects of AsA were detected in any of the maturation,

8 fertilization or embryo development parameters assessed. In experiment 2, we evaluated

9 the effects of adding AsA to vitrification-warming media on the post-warming survival

10 and quality of blastocysts. Day-6 in vitro-produced blastocysts $(\mathrm{N}=588)$ from six

11 replicates were randomly divided in two groups, with vitrification and warming media

12 either supplemented with $50 \mu \mathrm{g} / \mathrm{mL}$ AsA (VW+ group) or un-supplemented (VW-

13 control). Addition of AsA increased $(\mathrm{P}<0.05)$ blastocyst survival rate after vitrification

14 compared with that of VW- control embryos. Vitrification and warming increased

$15(\mathrm{P}<0.05)$ the production of oxygen species $(\mathrm{ROS})$ and reduced $(\mathrm{P}<0.05)$ the glutathione

16 levels in blastocysts. Although VW+ blastocysts displayed higher $(\mathrm{P}<0.05)$ ROS levels

17 than those of fresh control blastocysts, the levels were lower $(\mathrm{P}<0.05)$ than those found

18 in VW- control blastocysts. In conclusion, under the experimental conditions,

19 supplementation of IVM/IVF/IVC media with AsA did not improve the embryo

20 production in vitro. By contrast, the addition of AsA to chemically defined vitrification

21 and warming media increased the survival of in vitro-produced porcine blastocysts by

22 decreasing ROS production.

24 Keywords: Ascorbic acid; porcine; in vitro maturation; in vitro fertilization; 25 vitrification. 


\section{Introduction}

2 In vitro production (IVP) of porcine embryos is an important tool for agricultural,

3 biotechnological and biomedical purposes. Although substantial progress has been

4 achieved in embryo IVP systems [1,2,3], the overall efficiency remains unsatisfactory

5 because of the prevailing high incidence of polyspermy during IVF [4-7], leading to low

6 efficiency of blastocyst production and to poor quality of the resulting blastocysts $[8,9]$.

7 Although many different supplements, such as follicular fluid [10], vitamins [11,12],

8 growth factors [9] or hormones [13], have been added to IVP media in attempts to

9 improve the IVP of porcine embryos, the general assumption is that IVC conditions

10 remain suboptimal. One of the primary differences between in vitro and in vivo

11 conditions is related to oxidative stress [14]. The oxygen content of IVP environments

12 is higher than that in vivo, which results in increased production of ROS $[15,16]$. These

13 high ROS levels during embryo IVP are harmful to gametes and embryos [17-19]. Thus,

14 protecting oocytes and embryos against oxidative stress during in vitro culturing is a

15 key step for improving embryo IVP efficiency and embryo quality. To achieve this goal,

16 a widely used strategy is the addition of antioxidants to the media [12,20-23].

17 Ascorbic acid (AsA), the most important antioxidant in extracellular fluids [24], is one

18 of the antioxidants tested in embryo IVP that shows some beneficial properties. When

19 present during IVC, AsA increases the cleavage rate in bovine [25] and blastocyst rates

20 in ovine [26]. In porcine, AsA shows positive effects on oocyte nuclear maturation [12]

21 and blastocyst formation after parthenogenetic activation [27]. Addition of AsA also

22 apparently protects embryos against oxidative stress during IVC, improving embryo

23 developmental competence after either IVF [28] or parthenogenesis [27,29]. In some

24 studies, the quality of blastocysts in terms of cell numbers [29] or survival after

25 vitrification [30] also increases with supplementation with AsA during IVC. Moreover, 
1 addition of AsA to vitrification and warming media supplemented with serum increases

2 embryo survival rates of in vitro-produced porcine blastocysts [30].

3 Collectively, the above studies suggest that AsA could be an interesting molecule to use

4 during embryo IVP and/or vitrification in porcine to avoid the excessive increase of

5 ROS and their deleterious effects on oocytes and embryos. However, no systematic

6 study has explored the influence of AsA on each step of the embryo IVP system and the

7 presence of possible synergistic effects. Therefore, the aims of the present study were

8 the following: (1) evaluate the effects of AsA supplementation to IVM, IVF and IVC

9 media, including all possible combinations, on maturation, fertilization and embryonic

10 developmental parameters; and (2) assess the effects of adding AsA to vitrification and

11 warming defined media on the vitrification survival of IVP-porcine blastocysts.

13 2. Materials and methods

14 All experimental procedures used in this study were performed in accordance with the 15 2010/63/EU EEC Directive for animal experiments and were reviewed and approved by 16 the Ethical Committee for Experimentation with Animals of the University of Murcia, 17 Spain (research code: 1002/2012).

\subsection{Reagents and culture media}

20 Unless otherwise specified, all chemicals used in this study were purchased from

21 Sigma-Aldrich Co. (Alcobendas, Madrid, Spain). A physiological saline solution

22 composed of $\mathrm{NaCl} 0.9 \%(\mathrm{w} / \mathrm{v})$ and $70 \mu \mathrm{g} / \mathrm{mL}$ kanamycin was used to transport ovaries

23 from the slaughterhouse to the laboratory. The cumulus-oocyte complexes (COCs)

24 were collected and washed in Tyrode’s lactate supplemented with 10-mM HEPES and

25 0.1\% (w:v) polyvinyl alcohol (TL-PVA) [31,32]. The oocyte maturation medium was 
1 TCM-199 (Gibco Life Technologies S.A., Barcelona, Spain) supplemented with 0.57-

$2 \mathrm{mM}$ cysteine, $0.1 \%$ (w:v) PVA, $10 \mathrm{ng} / \mathrm{mL}$ EGF, $75 \mu \mathrm{g} / \mathrm{mL}$ penicillin G potassium, and

$350 \mu \mathrm{g} / \mathrm{mL}$ streptomycin sulfate. The basic medium used for IVF was a modified Tris-

4 buffered medium [33] enriched with 2.0-mM caffeine and $0.2 \%$ (w:v) BSA. A

5 Dulbecco's PBS (Gibco, Grand Island, NY) with $4 \mathrm{mg} / \mathrm{mL}$ of BSA was used for

6 washing spermatozoa after thawing and before re-suspension in IVF medium. The

7 embryo culture medium was North Carolina State University 23 (NCSU23)

8 [34] supplemented with $0.4 \%$ BSA. The basic medium for vitrification and warming

9 was the chemically defined TL-PVA medium. The first vitrification medium (V1) was

10 TL-PVA containing 7.5\% (v:v) of ethylene glycol and 7.5\% of dimethyl sulfoxide

11 (DMSO), and the second vitrification medium (V2) was TL-PVA containing 16\% (v:v)

12 of ethylene glycol and 16\% DMSO and 0.4 M sucrose. The warming medium consisted

13 of TL-PVA supplemented with $0.13 \mathrm{M}$ sucrose.

\subsection{Cumulus-oocyte complexes (COCs) collection and in vitro maturation}

16 Ovaries were collected from pre-pubertal gilts at a local slaughterhouse and transported 17 to the laboratory at the University of Murcia at $35{ }^{\circ} \mathrm{C}$ within $1 \mathrm{~h}$ of collection. Then, medium-sized follicles (3-6 mm in diameter) were sectioned with a sterile scalpel blade into TL-PVA to collect COCs. Oocytes surrounded by two or more layers of compact cumulus cells and with evenly granulated cytoplasm were selected and washed in

21 maturation medium. Groups of 75-80 COCs were transferred into a well of a four-well 22 multidish (Nunc, Roskilde, Denmark) containing $500 \mu \mathrm{L}$ of maturation medium 23 supplemented with $10 \mathrm{IU} / \mathrm{mL}$ eCG (Folligon; Intervet International B.V., Boxmeer, The 24 Netherlands) and 10 IU/mL hCG (Veterin corion; Divasa Farmavic S.A., Barcelona, 25 Spain) for $22 \mathrm{~h}$. The oocytes were then incubated for another 20 to $22 \mathrm{~h}$ in maturation 
1 medium without hormones. Maturation was performed under mineral oil at $38.5^{\circ} \mathrm{C}$ in

$25 \% \mathrm{CO}_{2}$ in air and $95 \%$ to $97 \%$ relative humidity.

3

\section{$4 \quad$ 2.3. In vitro fertilization}

5 After oocyte maturation, cumulus cells were removed with $0.1 \%$ hyaluronidase in 6 maturation medium by vortexing for $2 \mathrm{~min}$ at 1,660 rounds/min. The denuded oocytes 7 were washed three times in IVM medium and three times in IVF medium. Then, groups 8 of 30 oocytes were placed into $50 \mu \mathrm{L}$ drops in IVF medium in a $35 \mathrm{~mm} \times 10 \mathrm{~mm}$ Petri 9 dish (Falcon; Becton Dickinson Labware, Franklin Lakes, NJ, USA) under mineral oil 10 and were maintained in an incubator $\left(38.5^{\circ} \mathrm{C}, 5 \% \mathrm{CO}_{2}\right.$ in air and $95 \%$ to $97 \%$ relative

11 humidity) until addition of spermatozoa. Semen from a mature boar cryopreserved in $120.5 \mathrm{~mL}$ straws as described by Roca et al. [35] was thawed (two straws per replicate) in 13 a circulating water bath at $37^{\circ} \mathrm{C}$ for $20 \mathrm{~s}$. Immediately after thawing, $100 \mu \mathrm{L}$ of semen 14 was washed three times by centrifugation at $\times 1,900 \mathrm{~g}$ for $3 \mathrm{~min}$ in $10 \mathrm{~mL}$ of Dulbecco’s 15 PBS. The resulting pellet was suspended in IVF medium. After appropriate extension, $1650 \mu \mathrm{L}$ of this sperm suspension was added to the medium with the oocytes such that the 17 final sperm concentration was $3 \times 10^{5}$ spermatozoa/mL; thus, each oocyte was exposed 18 to 1,000 spermatozoa. Oocytes were co-incubated with spermatozoa for $5 \mathrm{~h}$ under 19 mineral oil at $38.5{ }^{\circ} \mathrm{C}$ in an atmosphere of $5 \% \mathrm{CO}_{2}$ in air and $95 \%$ to $97 \%$ relative 20 humidity.

\section{2.4. In vitro culture and assessment of embryonic development}

23 After gamete co-incubation, presumptive zygotes were washed by three rounds of 24 mechanical pipetting in IVC medium to remove spermatozoa that were not bound to the 25 zona pellucida. Presumptive zygotes were then transferred (30 zygotes per well) into a 
1 four-well multidish containing $500 \mu \mathrm{L}$ of glucose-free embryo culture medium

2 (NCSU23-BSA) supplemented with $0.3-\mathrm{mM}$ pyruvate and 4.5-mM lactate for 2 days

3 and then changed to fresh embryo culture medium containing 5.5-mM glucose for an

4 additional 5 day period. Embryo culture was performed under paraffin oil in an

5 incubator at $38.5{ }^{\circ} \mathrm{C}, 5 \% \mathrm{CO} 2$ in air, and $95 \%$ to $97 \%$ relative humidity.

6

7 2.5. Assessment of maturation, fertilization and embryo development

8 To evaluate the maturation and fertilization, representative aliquots of oocytes and

9 presumptive zygotes were fixed at $44 \mathrm{~h}$ of IVM and at $18 \mathrm{~h}$ after IVF, respectively, in a

10 solution of acetic acid:ethanol (1:3) for $72 \mathrm{~h}$ at room temperature. Fixed oocytes and

11 presumptive zygotes were stained with $1 \%(\mathrm{w}: \mathrm{v})$ lacmoid in $45 \%$ acetic acid and

12 examined under a phase-contrast microscope at magnification $\times 400$. Oocytes with

13 chromatin enclosed in a nuclear membrane or those with condensed chromatin but

14 without extruded polar body were classified as immature oocytes. Oocytes were

15 considered mature when their chromosomes were organized at metaphase and they

16 showed an extruded first polar body (MII).

17 Presumptive zygotes were considered penetrated when they contained one or more 18 swollen sperm heads and/or male pronuclei and two polar bodies. Sperm penetration

19 rate was the ratio of the number of penetrated oocytes relative to the total number of

20 mature oocytes inseminated. Monospermic rate was calculated as the ratio of oocytes

21 with one female pronucleus, one male pronucleus and two polar bodies to the total

22 number of matured oocytes penetrated. The efficiency of fertilization was the ratio of

23 the number of monospermic oocytes relative to the total number of matured oocytes

24 inseminated. 
1 The cleavage (ratio of the number of embryos cleaved to two to four cells of the total

2 number of oocytes inseminated) and blastocyst formation rates (ratio of the number of

3 blastocysts of the total number of cleaved embryos) were morphologically evaluated

4 using a stereomicroscope at Day 2 and Day 7 post-insemination (Day 0), respectively.

5 The total efficiency was described as the percentage of the total number of inseminated

6 oocytes that reached the blastocyst stage. Blastocysts were fixed in $4 \%(\mathrm{v}: \mathrm{v})$

7 paraformaldehyde in PBS for $30 \mathrm{~min}$ at room temperature $\left(24^{\circ} \mathrm{C}\right)$ to assess total cell

8 numbers. After fixation, embryos were washed with PBS containing $3 \mathrm{mg} / \mathrm{mL}$ BSA

9 (PBS-BSA), placed on a slide in a drop of $4 \mu \mathrm{L}$ of VECTASHIELD (Vector Labs,

10 Burlingame, CA, USA) containing $10 \mu \mathrm{g} / \mathrm{mL}$ Hoechst 33342, and covered with a

11 coverslip. Stained embryos were examined with fluorescence microscopy using a 330 to

12 380-nm excitation filter. The total number of nuclei that showed blue fluorescence was

13 counted.

\subsection{Vitrification and warming; assessment of survival and hatching rates}

16 Vitrification was performed according to the method described previously [36,37]. All

17 media used for vitrification and warming were held at $38.5^{\circ} \mathrm{C}$. Briefly, groups of five to

18 six embryos were washed twice in TL-PVA and consecutively equilibrated in V1 for 3

19 min and in V2 for 1 min for vitrification. During the final equilibration, embryos were

20 placed in a $1 \mu \mathrm{L}$ drop and loaded in the narrow end of a super open pulled straw (SOPS;

21 Minitüb, Tiefenbach, Germany) by capillary action. Subsequently, straws containing the

22 embryos were horizontally plunged into liquid nitrogen $\left(\mathrm{LN}_{2}\right)$. After storage in $\mathrm{LN}_{2}$ for

23 one week, the straws were removed and warmed by the one-step warming method

$24[38,39]$. Briefly, the straws were vertically submerged in one well of a four-well

25 multidish containing $800 \mu \mathrm{L}$ of warming medium and equilibrated for $5 \mathrm{~min}$. Then, 
1 vitrified-warmed blastocysts were washed in TL-PVA and cultured in vitro to assess the

2 embryo viability. Vitrified-warmed blastocysts that reformed their blastocoelic cavities

3 after warming and displayed a normal or thinning zona pellucida with an excellent or

4 good appearance during the culture were considered viable. The survival rate was

5 defined as the ratio of viable embryos to the total number of vitrified-warmed cultured

6 blastocysts. Additionally, after warming, the hatching rate (ratio of hatching or hatched

7 embryos at the end of the culture to the total number of cultured embryos) was

8 evaluated.

\section{$10 \quad$ 2.7. Differential staining}

11 The number of inner cell mass (ICM) and trophectoderm (TE) cells of the vitrified12 warmed blastocysts was determined using an indirect immunofluorescence protocol 13 [39]. Blastocysts were fixed with paraformaldehyde as described before for total cell 14 evaluation. Fixed embryos were permeabilized (1.5\% Triton X-100 and 0.15\% Tween 1520 in PBS) at $4{ }^{\circ} \mathrm{C}$ overnight. Permeabilized blastocysts were incubated at room 16 temperature first in a 2 N HCL solution for $20 \mathrm{~min}$ and then in $100 \mathrm{mM}$ Tris-HCl $(\mathrm{pH}$ 17 8.5) for 10 min. After denaturation and washing (3 times for 2 min in PBS-BSA), 18 blastocysts were incubated for $6 \mathrm{~h}$ in blocking solution (PBS containing 1\% BSA, 10\% 19 Normal Donkey Serum and $0.005 \%$ Tween 20 ) at $4{ }^{\circ} \mathrm{C}$. After washing (3 times for 2 20 min in PBS-BSA), blastocysts were incubated with the primary CDX-2 antibody (1:200 21 in the commercial dilution solution; BioGenex, San Ramon, CA, USA) for 1.5 days. 22 Then, blastocysts were washed (3 times for 2 min in PBS-BSA) and incubated with 23 donkey anti-mouse IgG-Alexa Fluor ${ }^{\circledR} 568$ conjugate (1:1000 in blocking solution; 24 Invitrogen, Rockford, USA). Finally, blastocysts were washed twice for 15 min in PBS25 BSA, placed on a slide in $4 \mu \mathrm{L}$ of Vectashield (Vector Labs, Burlingame, CA, USA) 
1 containing $10 \mu \mathrm{g} / \mathrm{mL}$ Hoechst 33342, and covered with a coverslip. Stained blastocysts

2 were examined with a fluorescence microscope using an excitation wavelength of 330-

3 to $380-\mathrm{nm}$ to count the total number of nuclei stained with Hoechst and a $536 \mathrm{~nm}$

4 excitation filter to count trophectoderm cells that were stained with Alexa Fluor 568

5 showing red fluorescence.

6

7 2.8. Measurement of intracellular GSH and ROS levels

8 Intracellular GSH and ROS levels of embryos were determined by staining with

9 CellTracker Blue (4-chloromethyl-6.8-difluoro-7-hydroxycoumarin; CMF2HC;

10 Invitrogen, ThermoFisher scientific, Massachusetts, USA) and H2DCFDA (2', 7'-

11 dichlorodihydrofluorescein diacetate; Invitrogen), respectively. Blastocysts were

12 washed in TL-PVA and incubated in the dark for $30 \mathrm{~min}$ in TL-PVA medium

13 containing $10 \mu \mathrm{M}$ CellTracker Blue and $10 \mu \mathrm{M}$ H2DCFDA at $38.5^{\circ} \mathrm{C}$. Stained embryos

14 were washed three times in TL-PVA. Then, groups of 5 embryos were transferred into

$15 \quad 10-\mu \mathrm{L}$ droplets of TL-PVA medium, and the fluorescence was immediately observed

16 with fluorescence microscopy with UV filters (370 nm for GSH and $460 \mathrm{~nm}$ for ROS).

17 Fluorescence emissions were recorded as TIFF files using a digital camera connected to

18 the fluorescent microscope. The fluorescence intensities of each blastocyst were

19 analyzed by ImageJ software (Version 1.51h; National Institutes of Health, Bethesda,

20 MD, USA).

21

22 2.9. Experimental design

23

24

2.9.1. Experiment 1 
1 In the first experiment, the effects of AsA supplementation in IVM, IVF and IVC media

2 on maturation, fertilization and embryo development were evaluated. For the

3 evaluation, oocyte maturation, fertilization, and embryo culture were performed in the

4 presence or absence of $50 \mu \mathrm{g} / \mathrm{mL}$ AsA in all possible combinations, which involved a

5 total of 8 experimental groups. A total of 2,744 oocytes were used in six replicates. A

6 random subset of oocytes $(\mathrm{N}=149)$ and presumed zygotes $(\mathrm{N}=1,142)$ from each group

7 was fixed and stained at $44 \mathrm{~h}$ of IVM and at $18 \mathrm{~h}$ after IVF to evaluate the maturation

8 and fertilization parameters, respectively. The remaining presumptive zygotes

$9 \quad(\mathrm{~N}=1,602)$ were cultured to assess in vitro embryo development. Day 7 blastocysts were

10 fixed and stained to assess their total cell number.

\subsubsection{Experiment 2}

13 In the second experiment, the effect of adding AsA to vitrification-warming media on

14 the post-warming survival and quality of in vitro-produced porcine blastocysts was

15 evaluated. The IVP of blastocysts was performed without AsA. This experiment was

16 performed in a total of six replicates. Day-6 in IVP full-expanded blastocysts $(\mathrm{N}=588)$,

17 morphologically classified as excellent or good, were randomly divided into one of two

18 groups in which vitrification and warming media were supplemented with $50 \mu \mathrm{g} / \mathrm{mL}$

19 AsA (VW+ group) or not supplemented (VW- control). This AsA concentration was

20 selected based on previous experiments (Kere et al., 2013). After warming, VW+

$21(\mathrm{~N}=281)$ and VW- control $(\mathrm{N}=307)$ blastocysts were cultured in vitro for $24 \mathrm{~h}$ to assess

22 the embryo survival and hatching rates. A random subset $(\mathrm{N}=21)$ of vitrified-warmed

23 blastocysts classified as viable from each group were subjected to differential staining to

24 assess the total number of cells, the number of cells in the inner cell mass (ICM) and the

25 number of cells in the trophectoderm (TE). Finally, the intracellular GSH and ROS 
1 levels from $\mathrm{VW}+$, VW- control and some fresh in vitro-produced blastocysts (Fresh

2 control) were measured.

3

\section{$4 \quad 2.10$. Statistical analyses}

5 Statistical analysis was performed using the IBM SPSS 19 statistical software package

6 (SPSS, Chicago, IL, USA). Continuous variables are expressed as the mean \pm SD.

7 Binary variables (maturation, penetration, monospermy, cleavage, blastocyst formation,

8 efficiency, survival and hatching rates) were obtained by calculating the percentage in

9 every well of each experimental group and in each of the replicates. These data are

10 expressed as the mean \pm standard deviation (SD). The Kolmogorov-Smirnov test was

11 used to test for normally distributed data. Means of more than two groups were

12 compared using a mixed-model analysis of variance (ANOVA), followed by the

13 Bonferroni post hoc test. Pairwise comparisons of means were performed using

14 Student's t-test. Differences were considered significant at $\mathrm{P}<0.05$.

16 3. Results

$18 \quad$ 3.1. Experiment 1.

19 Effects of AsA supplementation on IVM, fertilization and embryo development.

20 The addition of AsA had no effect on the percentage of MII oocytes at $44 \mathrm{~h}$ of 21 maturation between treatment (81.4\%) and control (83.5\%). Supplementation of IVM, 22 IVF and IVC media also had no effect on any fertilization parameter (Table 1). Rate of 23 sperm penetration was close to $70 \%$ with monospermy approximately $60 \%$ in all 24 groups. The overall IVF efficiency of the IVP system ranged from $37.6 \pm 8.5 \%$ to $2547.3 \pm 13.9 \%$ with no differences between treatment and control groups. 
1 The embryonic development parameters are presented in Table 2. Addition of AsA did

2 not affect the development to the 2-4 cell stage or blastocyst formation at the end of the

3 culture period. The total efficiency of blastocyst production was always approximately

$430 \%$. The quality of the in vitro-produced blastocysts in terms of total cell number

5 (range from $44.1 \pm 20.4$ to $53.0 \pm 26.2$ cells) did not vary with AsA supplementation.

6

\section{3.2. Experiment 2}

8 Effects of AsA supplementation to vitrification and warming media on blastocyst

9 survival, hatching rate and embryo quality.

10 The addition of AsA during vitrification and warming increased $(\mathrm{P}<0.05)$ blastocyst

11 survival rate compared with that of VW- control embryos (Table 3). However, the

12 blastocysts survival and hatched rates $24 \mathrm{~h}$ after warming were not affected by AsA

13 addition. Total cell numbers and the distribution of cells between the TP and the ICM

14 (Table 4) were also comparable between the two vitrification groups.

15 The vitrification and warming procedures increased $(\mathrm{P}<0.05)$ intracellular ROS and

16 decreased $(\mathrm{P}<0.05)$ GSH levels, compared with those of the controls (Fig. 1). Addition

17 of AsA to the vitrification-warming media decreased $(\mathrm{P}<0.05)$ ROS production but did 18 not affect GSH. Those embryos vitrified and warmed without AsA (VW- control)

19 displayed the highest $(\mathrm{P}<0.05)$ intracellular ROS values, whereas those treated with

20 AsA had intermediate ROS levels.

\section{4. Discussion}

23 In the present study, the effects of AsA treatment were assessed during porcine IVM,

24 IVF and/or IVC on variables of maturation, fertilization and embryonic development.

25 Despite previous reports of some positive effects of exogenous antioxidants within IVP, 
1 the present study showed that the supplementation of media with AsA did not improve

2 the overall efficiency of our IVP system. However, AsA had a clear beneficial effect

3 during vitrification and warming, increasing the vitrification survival of in vitro-

4 produced blastocysts.

5 In the current study, AsA supplementation to oocyte IVM medium did not significantly

6 affect the maturation or fertilization rates or the subsequent embryo development after

7 IVF. These results are consistent with previous investigations in ovine [26], bovine [39]

8 and porcine [11], which did not demonstrate any effect of AsA supplementation during

9 IVM on the developmental competence of porcine oocytes and embryos. Some studies

10 report positive effects of AsA during maturation using parthenogenetically activated

11 [27] or denuded oocytes [12]. Kere et al. [27] observed an increase of the cleavage rate,

12 blastocyst formation and blastocyst total cell number after parthenogenetic activation of

13 porcine oocytes matured with AsA. The effect of AsA on parthenogenetically activated

14 oocytes could be due to the higher sensitivity of these oocytes to oxidative

15 environments than those oocytes subjected to IVF [40]. Consistent with this hypothesis,

16 Tao et al. [12] demonstrated improved nuclear maturation of porcine denuded oocytes

17 when AsA was added to the IVM medium. In this case, AsA during IVM was also

18 beneficial under an increased oxidative stress condition due to the absence of cumulus

19 cells surrounding the oocytes during IVM [41]. Together with our present results, these

20 previous observations suggest that AsA has a beneficial effect during maturation when

21 the generation of ROS is extremely high or protective mechanisms against oxidative

22 stress are lacking.

23 We believe this is the first study to evaluate the effects of the addition of AsA during

24 IVF of porcine oocytes. Previous studies on the effects of antioxidants during the IVF

25 period have reported contradictory results. With regard to IVF, it is important to note 
1 that physiological levels of ROS are required to induce hyperactivation and capacitation

2 of spermatozoa [42,20], both pre-requisites for fertilization. Although some antioxidants

3 added to sperm before or during IVF have improved subsequent developmental capacity

4 of bovine and ovine embryos [43], excess antioxidants impair fertilization, normal

5 pronuclear formation and embryo development in bovine [44]. In our study, the

6 supplementation of IVF media with AsA did not have any effect on the fertilization

7 parameters or on the developmental competence of fertilized oocytes.

8 With respect to the embryo culture, the results followed the same pattern as those for

9 IVM and IVF. Thus, the addition of AsA to the IVC medium did not alter the IVC

10 parameters and did not affect the number of cells in the blastocysts. These observations

11 are similar to those reported by Castillo-Martín et al. [30]; however, they are in contrast

12 to Hossein et al. [28] who obtained increased blastocyst formation when the ICV

13 medium was enriched with AsA. To understand these discrepancies, we should consider

14 the overall efficiency of each IPV system. Thus, our total blastocyst production

15 efficiency (30-35\%) and that reported by Castillo-Martín et al. [30] (20\%) are

16 considered adequate for a porcine IVP system, and in these circumstances, AsA did not

17 exert any effect. By contrast, under less efficient conditions in which the blastocyst

18 formation rate was close to $9 \%$ [28], the addition of AsA was beneficial. A positive

19 effect of AsA was also observed with embryos derived from parthenogenesis [27,29],

20 which may be more sensitive to oxidative stress than IVF-derived embryos because of

21 their biological differences [45]. It is possible that AsA only exerts positive effects

22 under highly oxidant conditions in which the detrimental effects of ROS are

23 exacerbated. Following this logic, the present study showed a positive effect of AsA

24 during vitrification and warming using chemically defined media, and these results are

25 consistent with those obtained by Castillo-Martín et al. [30] using a vitrification and 
1 warming system based on supplementation with fetal serum and AsA. During

2 vitrification and warming, embryos are subjected to important disturbances in the redox

3 status due to an increase of ROS and/or a decrease in the GSH levels [30,46,47]. In this

4 study, vitrification and warming increased, as expected, the oxidative stress altering

5 intracellular GSH and ROS levels compared with those of fresh blastocysts. However,

6 addition of AsA mitigated this high oxidative stress by reducing intracellular ROS

7 production of blastocysts and increasing the embryo survival rate after warming.

8 Supplementation of AsA to cryopreservation media of mouse and bovine embryos had

9 also beneficial effects by reducing peroxidation and increasing cryotolerance [48,49].

10 These results indicate that the addition of AsA or other antioxidants during vitrification

11 and warming could be an efficient strategy to reduce the oxidative stress related to this

12 technology and therefore to improve embryo survival.

13 In conclusion, under our experimental conditions and within a highly efficient porcine

14 in vitro embryo production system, the supplementation of IVM/IVF/IVC media with

15 AsA at a concentration of $50 \mu \mathrm{g} / \mathrm{mL}$ failed to further increase the IVP-outcomes. By

16 contrast, the addition of AsA to chemically defined vitrification and warming media

17 increased the vitrification survival by decreasing the ROS production. Thus, AsA

18 supplementation is recommended for vitrification and particularly for unstable or low

19 performing IVP systems.

\section{Acknowledgments}

22 The authors are grateful to Moises Gonzalvez for his assistance throughout this work.

23 We thank the Ministry of Economy and Competitiveness (Madrid, Spain) for its grant-

24 based support of A Nohalez and CA Martinez (BES-2013-064069 and BES-2013-

25 064087, respectively). 


\section{$1 \quad$ Funding}

2 This study was supported by the Ministry of Economy and Competitiveness (Madrid,

3 Spain)/the European Regional Development Fund (grant number AGL2015-69735-R),

4 and the Seneca Foundation, Murcia, Spain (grant number 19892/GERM/15).

6 Role of the funding source

7 Funding sources did not have any involvement in the study design, in the collection,

8 analysis and interpretation of data, in the writing of the report, and in the decision to

9 submit the article for publication.

11 Author contributions

12 A Nohalez, H Rodriguez-Martinez, EA Martinez and C Cuello conceived, designed and 13 directed the study. A Nohalez, CA Martinez, I Parrilla, MA Gil, EA Martinez and C 14 Cuello performed the experiments. A Nohalez, EA Martinez and C Cuello analyzed and 15 interpreted the data. A Nohalez, EA Martinez and C Cuello wrote the manuscript. J 16 Roca and H Rodriguez-Martinez critically revised the manuscript. All authors approved 17 the manuscript for publication.

\section{Declaration of interest}

20 None of the authors have any conflicts of interest to declare.

\section{References}

23 [1] Gil MA, Cuello C, Parrilla I, Vazquez JM, Roca J, Martinez EA. Advances in swine in vitro embryo production technologies. Reprod Domest Anim 2010;45:40-8. 
1 [2] Zhang $\mathrm{W}$, Yi K, Yan $\mathrm{H}$, Zhou $\mathrm{X}$. Advances on in vitro production and

3 [3] Grupen CG. The evolution of porcine embryo in vitro production. Theriogenology 2014;81:24-37.

[4] Abeydeera. LR. In vitro production of embryos in swine. Theriogenolgy 2002;57:256-73.

[5] Romar R, Funahashi H, Coy P. In vitro fertilization in pigs: New molecules and protocols to consider in the forthcoming years. Theriogenology 2016;85:125-34.

[6] Martinez CA, Nohalez A, Cuello C, Vazquez JM, Roca J, Martinez EA, et al. The use of mineral oil during in vitro maturation, fertilization, and embryo culture does not impair the developmental competence of pig oocytes. Theriogenology 2015;83:693-702.

[7] Martinez CA, Nohalez A, Ceron JJ, Rubio CP, Roca J, Cuello C, et al. Peroxidized mineral oil increases the oxidant status of culture media and inhibits in vitro porcine embryo development. Theriogenology 2017;103:17-23.

[8] Kikuchi K. Developmental competence of porcine blastocysts produced in vitro. J Reprod Dev 2004;50:21-8.

[9] Dang-Nguyen NT, Lo NW, Chuang SP, Jian YL, Ju JC. Sonic hedgehog supplementation of oocyte and embryo culture media enhances development of IVF porcine embryos. Reproduction 2011;142:87-97.

[10] Ito M, Iwata H, Kitagawa M, Kon Y, Kuwayama T, Monji Y. Effect of follicular fluid collected from various diameter follicles on the progression of nuclear maturation and developmental competence of pig oocytes. Anim Reprod Sci 2008;106:421-30. 
1 [11] Tatemoto H, Ootaki K, Shigeta K, Muto N. Enhancement of developmental competence after in vitro fertilization of porcine oocytes by treatment with ascorbic acid 2-O-alpha-glucoside during in vitro maturation. Biol Reprod 2001;65:1800-6.

[12] Tao Y, Zhou B, Xia G, Wang F, Wu Z, Fu M. Exposure to L-ascorbic acid or alpha-tocopherol facilitates the development of porcine denuded oocytes from metaphase I to metaphase II and prevents cumulus cells from fragmentation. Reprod Domest Anim 2004;39:52-7.

[13] Nam DH, Lee SH, Kim HS, Lee GS, Jeong YW, Kim S, et al. The role of gonadotropin-releasing hormone $(\mathrm{GnRH})$ and its receptor in development of porcine preimplantation preimplantation embryos derived from in vitro fertilization. Theriogenology 2005;63:190-201.

[14] Fischer B, Bavister BD. Oxygen tension in the oviduct and uterus of rhesus monkeys, hamster and rabbits. J Reprod Fertil 1993;99:673-9.

[15] Luvoni GC, Keskintepe L, Brackett BG. Improvement in bovine embryo production in vitro by glutathione-containing culture media. Mol Reprod Dev 1996;43:437-43.

[16] Takahashi M, Nagai T, Okamura N, Takahashi H, Okano A. Promoting effect of beta-mercaptoethanol on in vitro development under oxidative stress and cystine uptake of bovine embryos. Biol Reprod 2002;66:562-7.

[17] Luberda Z. The role of glutathione in mammalian gametes. Reproductive Biology 2005;5:5-17.

[18] Silva PF, Gadella BM, Colenbrander B, Roelen BA. Exposure of bovine sperm to pro-oxidants impairs the developmental competence of the embryo after the firstcleavage. Theriogenology 2007;67:609-19 
1 [19] Kim E, Jeon Y, Kim DY, Lee E, Hyun SH. Antioxidative effect of carboxyethylgermanium sesquioxide (Ge-132) on IVM of porcine oocytes and subsequent embryonic development after parthenogenetic activation and IVF. Theriogenology 2015;84:226-36

[20] Funahashi H. Effect of beta-mercaptoethanol during in vitro fertilization procedures on sperm penetration into porcine oocytes and the early development in vitro. Reproduction 2005;130:889-98.

[21] Nagai T, Funahashi H, Yoshioka K, Kikichi K. Update of in vitro production of porcine embryos. Front biosci 2006;11:2565-73.

[22] Gajda B. Factors and methods of pig oocyte and embryo quality improvement and their application in reproductive biotechnology. Reprod Biol 2009;9:97-112

[23] Kang JT, Kwon DK, Park SJ, Kim SJ, Moon JH, Koo OJ, et al. Quercetin improves the in vitro development of porcine oocytes by decreasing reactive oxygen species levels. J Vet Sci 2013;14:15-20

[24] Warren S, Patel S, Kapron CM. The effect of vitamin E exposure on cadmium toxicity in mouse embryo cells in vitro. Toxicology 2000;142:119-26.

[25] Córdova B, Morató R, Izquierdo D, Paramio T, Mogas T. Effect of the addition of insulin-transferrin-selenium and/or L-ascorbic acid to the in vitro maturation of prepubertal bovine oocytes on cytoplasmic maturation and embryo development. Theriogenology 2010;74:1341-8

[26] Natarajan R, Shankar MB, Munuswamy D. Effect of $\alpha$-tocopherol supplementation on in vitro maturation of sheep oocytes and in vitro development of preimplantation sheep embryos to the blastocyst stage. J Assist Reprod Genet 2010;27:483-90. 
1 [27] Kere M, Siriboon C, Lo NW, Nguyen NT, Ju JC. Ascorbic acid improves the developmental competence of porcine oocytes after parthenogenetic activation and somatic cell nuclear transplantation. J Reprod Dev 2013;59:78-84.

[28] Hossein MS, Hashem MA, Jeong YW, Lee MS, Kim S, Kim JH, et al. Temporal effects of alpha-tocopherol and L-ascorbic acid on in vitro fertilized porcine embryo development. Anim Reprod Sci. 2007;100:107-17.

[29] Hu J, Cheng D, Gao X, Bao J, Ma X, Wang H. Vitamin C enhances the in vitro development of porcine pre-implantation embryos by reducing oxidative stress Reprod Domest Anim 2012;47:873-9

[30] Castillo-Martín M, Bonet S1, Morató R, Yeste M. Comparative effects of adding $\beta$-mercaptoethanol or L-ascorbic acid to culture or vitrification-warming media on IVF porcine embryos. Reprod Fertil Dev 2014;26:875-82

[31] Funahashi H, Nagai T. Sperm selection by a climbing-over-a-wall IVF method reduces the incidence of polyspermic penetration of porcine oocytes. J Reprod Dev 2000;46:319-24.

[32] Martinez EA, Angel MA, Cuello C, Sanchez-Osorio J, Gomis J, Parrilla I, et al. Successful non-surgical deep uterine transfer of porcine morulae after 24 hour culture in a chemically defined medium. PLoS One. 2014; 13:9:e104696.

[33] Abeydeera LR, Day BN. In vitro penetration of pig oocytes in a modified Trisbuffered medium: effect of BSA, caffeine and calcium. Theriogenology 1997;48:537-44.

[34] Petters RM, Wells KD. Culture of pig embryos. J Reprod Fertil Suppl 1993;48:61-73. 
1 [35] Roca J, Gil MA, Hernandez M, Parrilla I, Vazquez JM, Martinez EA. Survival and fertility of boar spermatozoa after freeze-thawing in extender supplemented with butylated hydroxytoluene. J Androl 2004;25:397-405.

[36] Berthelot F, Martinat-Botté F, Locatelli A, Perreau C, Terqui M. Piglets born after vitrification of embryos using the open pulled straw method. Cryobiology 2000;41:116-24.

[37] Cuello C, Sanchez-Osorio J, Almiñana C, Gil MA, Perals ML, Lucas X, et al.

[39] Dalvit G, Llanes SP, Descalzo A, Insani M, Beconi M, Cetica P. Effect of alphaEffect of the cryoprotectant, concentration on the in vitro embryo development and cell proliferation of OPS-vitrified porcine blastocysts. Cryobiology 2008;56:189-94.

[38] Sanchez-Osorio J, Cuello C, Gil MA, Almiñana C, Parrilla I, Caballero I, et al. Factors affecting the success rate of porcine embryo vitrification by the Open Pulled Straw method. Anim Reprod Sci 2008;108:334-44. tocopherol and ascorbic acid on bovine oocyte in vitro maturation Reprod Domest Anim 2005;40:93-7.

[38] Cuello C, Gil MA, Parrilla I, Tornel J, Vázquez JM, Roca J, et al. In vitro development following one-step dilution of OPS-vitrified porcine blastocysts. Theriogenology 2004;62:1144-52.

[39] Wydooge E, Vandaeke L, Beek J, Favoreel H, Heindryckx B, De Sutter P, et al. Differential apoptotic staining of mammalian blastocysts based on double immunofluorescent CDX2 and active caspase-3 staining. Anal Biochem 2011;416:228-30. 
1 [40] Booth PJ, Holm P, Callesen H. The effect of oxygen tension on porcine embryonic development is dependent on embryo type. Theriogenology 2005;63:2040-52.

[41] Trapphoff T, Heiligentag M, Simon J, Staubach N, Seidel T, Otte K, et al. Improved cryotolerance and developmental potential of in vitro and in vivo matured mouse oocytes by supplementing with a glutathione donor prior to vitrification. Mol Hum Reprod 2016;22:867-81.

[42] O'Flaherty CM, Beorlegui NB, Beconi MT. Reactive oxygen species requirements for bovine sperm capacitation and acrosome reaction. Theriogenology 1999;52:289-301.

[43] Pang YW, Sun YQ, Jiang XL, Huang ZQ, Zhao SJ, Du WH, et al. Protective effects of melatonin on bovine sperm characteristics and subsequent in vitro embryo development. Mol Reprod Dev 2016;83:993-1002.

[44] Gonçalves FS, Barretto LS, Arruda RP, Perri SH, Mingoti GZ. Effect of antioxidants during bovine in vitro fertilization procedures on spermatozoa and embryo development. Reprod Domest Anim 2010;45:129-35.

[45] Gomez E, Gutierrez-Adan A, Diez C, Bermejo-Alvarez P, Muñoz M, Rodriguez A, et al. Biological differences between in vitro produced bovine embryos and parthenotes. Reproduction 2009;137:285-95.

[46] Somfai T, Ozawa M, Noguchi J, Kaneko H, Kuriani Karja NW, Farhudin M, et al. Developmental competence of in vitro-fertilized porcine oocytes after in vitro maturation and solid surface vitrification: effect of cryopreservation on oocyte antioxidative system and cell cycle stage. Cryobiology 2007;55:115-26. 
1 [47] Martino NA, Dell'aquila ME, Cardone RA, Somoskoi B, Lacalandra GM, Cseh

S. Vitrification preserves chromatin integrity, bioenergy potential and oxidative parameters inmouse embryos. Reprod Biol Endocrinol 2013;11:27.

[48] Lane M, Maybach JM, Gardner DK. Addition of ascorbate during cryopreservation stimulates subsequent embryo development. Hum Reprod 2002; 17:2686-93.

[49] Higginbotham AR, Owen CM, Barceló-Fimbres M, Campos-Chillon LF. Effect of novel SOF medium and l-ascorbic acid during cryopreservation of in vitroproduced Jersey cattle embryos. Reprod Fertil Dev 2016; 29: 215-16. 


\section{Figure legends}

2

3 Fig. 1. Intracellular reactive oxygen species (ROS) and glutathione (GSH) levels of Day

46 porcine blastocysts vitrified-warmed in media without ascorbic acid (VW-control;

5 white bars) or in media supplemented with $50 \mu \mathrm{g} / \mathrm{mL}$ ascorbic acid (VW+ group; grey

6 bars). Non-vitrified Day 6 in vitro-produced blastocysts were also assessed (fresh

7 control; black bars). Different letters indicate significant differences among groups

$8 \quad(\mathrm{P}<0.01)$. Data are expressed as the mean \pm SD (six replicates).

9

10

11

12

13

14

15

16

17

18

19

20

21

22

23

24

25

26

27

28

29 


\section{Table 1}

2 Fertilization parameters of porcine oocytes cultured in media with $(+50 \mu \mathrm{g} / \mathrm{mL})$ or

3 without (-) ascorbic acid (AsA).

4

\begin{tabular}{|c|c|c|c|c|c|c|}
\hline \multicolumn{3}{|c|}{$\begin{array}{c}\text { AsA } \\
\text { supplementation }\end{array}$} & \multirow{2}{*}{$\begin{array}{c}\text { Oocytes } \\
(\mathrm{N})\end{array}$} & \multicolumn{2}{|c|}{ Oocytes (\%) } & \multirow{2}{*}{$\begin{array}{c}\text { Efficiency } \\
(\%)\end{array}$} \\
\hline IVM & IVF & IVC & & Penetrated & Monospermic & \\
\hline+ & + & + & 149 & $69.1 \pm 14.5$ & $71.2 \pm 10.1$ & $47.3 \pm 13.9$ \\
\hline+ & + & - & 123 & $63.5 \pm 13.9$ & $73.7 \pm 14.1$ & $43.2 \pm 5.2$ \\
\hline+ & - & + & 143 & $72.4 \pm 13.5$ & $60.9 \pm 18.1$ & $39.7 \pm 13.3$ \\
\hline+ & - & - & 130 & $73.0 \pm 11.2$ & $59.1 \pm 17.0$ & $40.0 \pm 8.9$ \\
\hline- & + & + & 161 & $63.3 \pm 10.9$ & $68.0 \pm 12.3$ & $37.6 \pm 8.5$ \\
\hline- & + & - & 148 & $78.3 \pm 14.7$ & $63.1 \pm 10.7$ & $45.1 \pm 8.1$ \\
\hline- & - & + & 141 & $72.0 \pm 5.4$ & $65.4 \pm 15.8$ & $42.0 \pm 10.2$ \\
\hline- & - & - & 147 & $72.4 \pm 15.3$ & $64.7 \pm 12.2$ & $46.3 \pm 5.7$ \\
\hline
\end{tabular}

5

6 Penetrated: Number of oocytes penetrated/total inseminated oocytes.

7 Monospermic: Number of oocytes with only one male pronucleus/total oocytes

8 penetrated.

9 Efficiency: Number of monospermic oocytes/total oocytes inseminated.

10 Data are expressed as the mean \pm SD (six replicates).

11

12

13

14

15

16

17

18

19

20 
Table 2

2 Embryonic development achieved after oocyte maturation, fertilization and embryo

3 culture with $(+, 50 \mu \mathrm{g} / \mathrm{mL})$ or without (-) ascorbic acid (AsA).

4

\begin{tabular}{|c|c|c|c|c|c|c|}
\hline \multicolumn{3}{|c|}{ AsA supplementation } & \multirow{2}{*}{$\begin{array}{c}\text { Oocytes } \\
\text { (N) }\end{array}$} & \multicolumn{3}{|c|}{ Embryonic development } \\
\hline IVM & IVF & IVC & & $\begin{array}{c}\text { Cleavage } \\
(\%)\end{array}$ & $\begin{array}{c}\text { Blastocyst } \\
(\%)\end{array}$ & $\begin{array}{c}\text { Total } \\
\text { efficiency } \\
(\%)\end{array}$ \\
\hline+ & + & + & 194 & $47.4 \pm 5.0$ & $73.8 \pm 19.1$ & $35.4 \pm 9.8$ \\
\hline+ & + & - & 200 & $45.4 \pm 7.5$ & $65.7 \pm 22.0$ & $29.5 \pm 10.4$ \\
\hline+ & - & + & 196 & $52.3 \pm 10.9$ & $67.1 \pm 16.3$ & $36.2 \pm 14.7$ \\
\hline+ & - & - & 188 & $49.1 \pm 12.9$ & $75.7 \pm 17.6$ & $37.5 \pm 13.6$ \\
\hline- & + & + & 202 & $47.9 \pm 14.5$ & $66.0 \pm 16.1$ & $32.1 \pm 13,5$ \\
\hline- & + & - & 200 & $45.9 \pm 9.9$ & $61.4 \pm 12.7$ & $27.7 \pm 10.3$ \\
\hline- & - & + & 199 & $51.6 \pm 8.9$ & $71.5 \pm 18.3$ & $37.2 \pm 12.2$ \\
\hline - & - & - & 223 & $47.4 \pm 5.0$ & $73.8 \pm 19.1$ & $35.4 \pm 9.8$ \\
\hline
\end{tabular}

5

6

7

8

9

10

11

12

13

14

15

16

17

18

19

20

21

22

23

24

Cleavage: Number of 2,4-cell embryos/total inseminated oocytes cultured.

Blastocyst: Number of blastocysts/total cleaved embryos.

Total Efficiency: Number of blastocysts/total inseminated oocytes cultured.

Data are expressed as the mean \pm SD (six replicates).

(1)

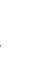




\section{$1 \quad$ Table 3}

2 Survival and hatching rates and quality (number and distribution of cells) after $24 \mathrm{~h}$ of

3 culturing of Day 6 in vitro-produced porcine blastocysts vitrified-warmed in media

4 supplemented with $50 \mu \mathrm{g} / \mathrm{mL}$ ascorbic acid (AsA; VW+) or without supplementation

5 (VW- control).

\begin{tabular}{ccccccc}
\hline & & Survival rate & Hatching & \multicolumn{3}{c}{ Cells in blastocysts } \\
\cline { 5 - 7 } Group & $\mathbf{N}$ & $(\mathbf{\%})$ & rate (\%) & $\begin{array}{c}\text { Total cell } \\
\text { number }\end{array}$ & TP $^{1}$ & ICM $^{2}$ \\
\hline VW+ & 307 & $51.1 \pm 20.9^{\mathrm{a}}$ & $10.7 \pm 12.0$ & $58.7 \pm 21.1$ & $44.2 \pm 18.2$ & $14.5 \pm 6.5$ \\
VW-control & 281 & $34.8 \pm 21.4^{\mathrm{b}}$ & $6.0 \pm 8.1$ & $62.6 \pm 14.4$ & $46.3 \pm 12.1$ & $16.7 \pm 7.5$ \\
\hline
\end{tabular}

6

$7 \quad{ }^{a, b}$ Different superscripts in the same column indicate a significant difference $(\mathrm{P}<0.05)$.

$8 \quad{ }^{1}$ TP: trophectoderm.

9 'ICM: inner cell mass.

10 Data are expressed as the mean \pm SD (six replicates).

11

12 


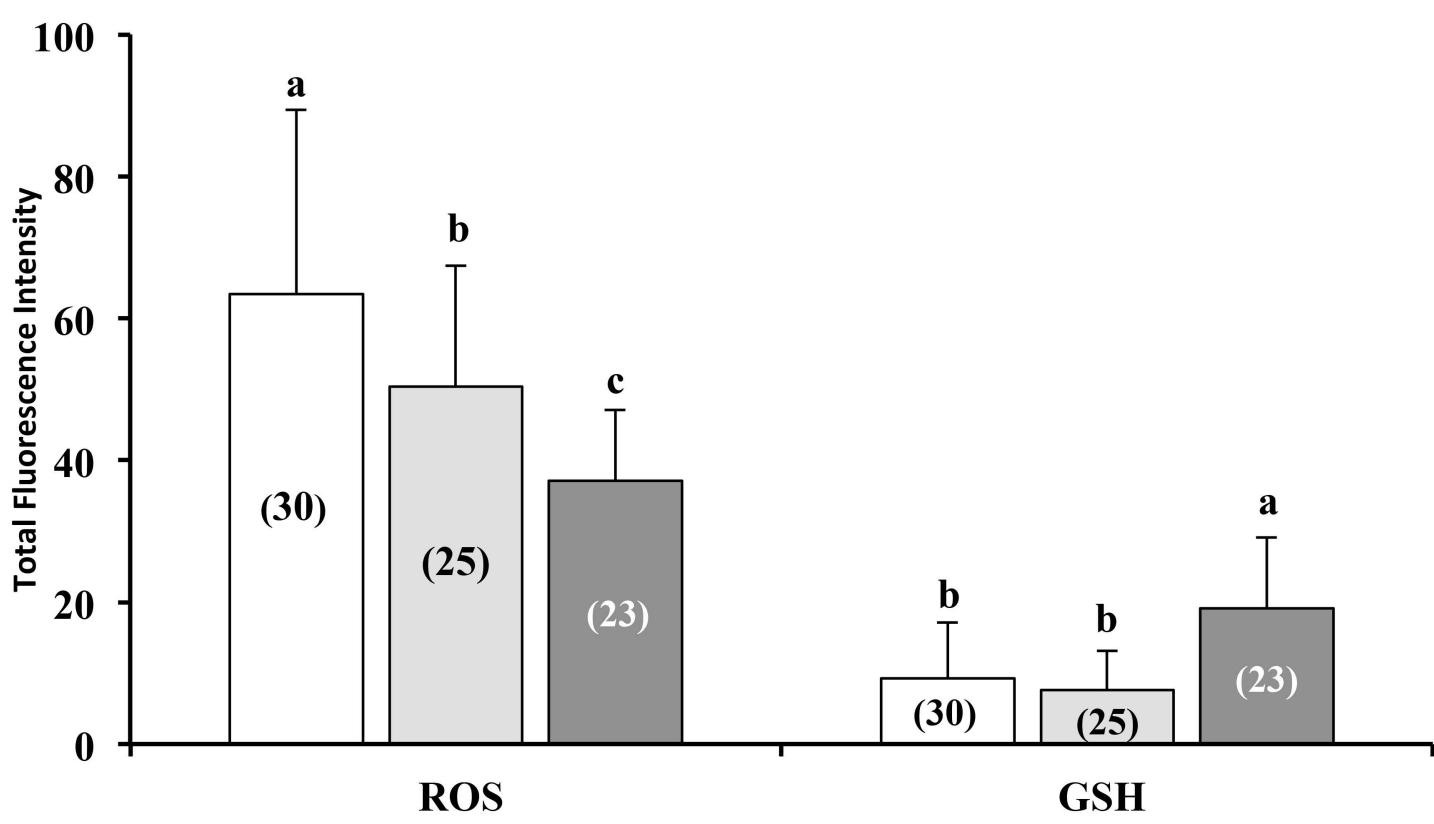

$\square$ VW Control $\quad \square$ VW+ group $\quad \square$ Fresh Control 